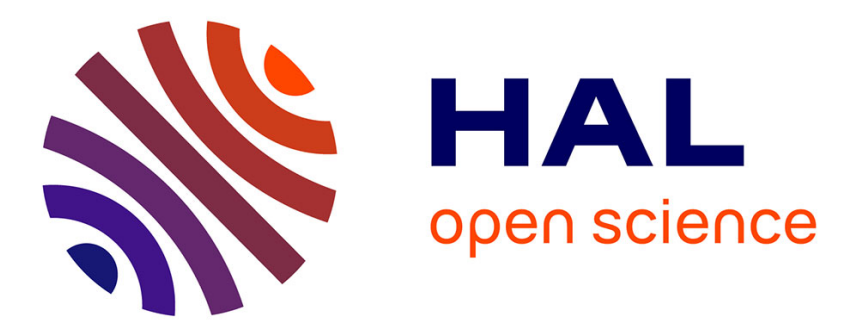

\title{
Maximum likelihood frequency estimation in smart grid applications
}

\author{
Vincent V. Choqueuse, El Houssin El Bouchikhi, Mohamed Benbouzid
}

\section{To cite this version:}

Vincent V. Choqueuse, El Houssin El Bouchikhi, Mohamed Benbouzid. Maximum likelihood frequency estimation in smart grid applications. ISIE 2015, Jun 2015, Buzios, Brazil. 10.1109/ISIE.2015.7281667 . hal-01212112

\section{HAL Id: hal-01212112 \\ https://hal.science/hal-01212112}

Submitted on 6 Oct 2015

HAL is a multi-disciplinary open access archive for the deposit and dissemination of scientific research documents, whether they are published or not. The documents may come from teaching and research institutions in France or abroad, or from public or private research centers.
L'archive ouverte pluridisciplinaire HAL, est destinée au dépôt et à la diffusion de documents scientifiques de niveau recherche, publiés ou non, émanant des établissements d'enseignement et de recherche français ou étrangers, des laboratoires publics ou privés. 


\section{Maximum Likelihood Frequency Estimation in Smart Grid Applications}

\author{
Vincent Choqueuse \\ University of Brest, EA 4325 LBMS \\ Rue de Kergoat, CS 93837 \\ 29238 Brest Cedex 03, France
}

\author{
Elhoussin Elbouchikhi \\ ISEN Brest, EA 4325 LBMS \\ 20 Rue Cuirass Bretagne, \\ 29200 Brest, France
}

\author{
Mohamed Benbouzid \\ University of Brest, EA 4325 LBMS \\ Rue de Kergoat, CS 93837 \\ 29238 Brest Cedex 03, France
}

\begin{abstract}
This paper focuses on the estimation of the fundamental frequency in balanced three-phase power systems. Specifically, we propose a Maximum Likelihood Estimator (MLE) that exploits the multidimensional nature of electrical signals. For perfectly sinusoidal signals, we show that the MLE can be expressed according to the periodogram of the instantaneous positive component. For harmonic signals, we demonstrate that the MLE can be approximated by a cumulated periodogram of the zero, positive and negative sequence components. As compared to single-phase estimators, statistical analysis and simulation results prove that the proposed estimator decreases the Mean Square Error by a factor of three, whatever the Signal to Noise Ratio (SNR) or data length. Furthermore, simulations with experimental data show that the proposed technique outperforms classical spectral estimators such as MUSIC.
\end{abstract}

Index Terms-Frequency Estimation, Power System, Smart Grid, Maximum Likelihood Estimator, Cramér-Rao Bounds

\section{INTRODUCTION}

The electrical network has undergone profound changes during the last decades. These changes are mainly due to two factors: the integration of renewable energy sources and the emergence of new consumer usages. Despite its ecological benefit, this mutation greatly complicates the management of the electricity grid. On one hand, renewable energy sources are intermittent and diffuse that make the energy generation hardly controllable and predictable. On the other, new usages such as the charging of Electric Vehicles are large energy consumers and make difficult to maintain the balance between energy production and consumption. To solve this problem, a promising solution relies on the use of Information and Communications Technology. The future "Smart Grid" is expected to be able to monitor and optimize its structure to maintain the balance between energy production and consumption.

In Smart-Grid, the electrical signal is measured and analyzed at substations with Phasor Measurement Units (PMUs) to monitor the grid state. A PMU is a device that is able to estimate several signal parameters from the current or voltage signals, such as the phasor or fundamental frequency. Among these parameters, the fundamental frequency plays a key role. Indeed, deviation from the nominal value can indicate a mismatch between production and consumption [1]. Furthermore, a large deviation can cause damage to equipment and should be detected at an early stage. For these reasons, frequency estimation is the backbone of smart grid monitoring.
Many frequency estimation techniques have been proposed [2], [3] and applied to electrical signals. These include the Discrete Fourier Transform [4], PLL [5], high resolution approaches [6], [7], Least square [8], and adaptive filtering [9]. Nevertheless, these techniques are general and do not take into account the particularities of electrical signals. From a statistical point of view, these particularities offer opportunities to improve the frequency estimation. Following this idea, several authors have developed parametric estimators specifically designed for electrical signals. A Least square estimators exploiting the harmonic structure of electrical signal has been described in [10], [11]. Another interesting feature of electrical signals is their multidimensional nature. Indeed, electrical signals are composed of three "phases" that can be used jointly for frequency estimation. Several techniques that exploit this property are available in the literature [12]-[20]. Most of them are based on Power electronic analysis tools such as the Clarke or Fortescue Transform. Despite their simplicity, these techniques have several limitations. First, these techniques assume perfect sinusoidal signals and their performance degrade drastically in the presence of harmonic components. Then, since these techniques focus on instantaneous frequency estimation, they may produce suboptimal estimators if the fundamental frequency is near constant over a period of time.

Despite this rich literature, there is no technique that jointly exploits the multidimensional and harmonic structure for frequency estimation. Furthermore, it is not clear how these particularities improve the performance of the frequency estimator. In this study, we address the fundamental frequency estimation problem for three-phase systems. Specifically, under the assumption of a balanced system, we propose a parametric frequency estimator that jointly exploits the multidimensional and harmonic structure of electrical signals. The contribution of this study is twofold: first, we derive the Maximum Likelihood Estimator (MLE) of the fundamental frequency, then we provide a statistical analysis aiming at measuring the benefit of using the multidimensional and harmonic structure. The remainder of the paper is organized as follows. Section II presents the signal model and the assumptions. Section III describes the MLE frequency estimator and Section IV provides an analysis of the Cramér-Rao Bounds. Finally, Section V reports on the performance of the proposed estimator with synthetic and experimental signals. 


\section{Signal Model}

In a three-phase system, voltage or current signals are composed of three components. Under balanced conditions, these components are phase-rotated from each other by $\frac{2 \pi}{3}$. In the ideal case, each component is a sinusoidal signal with (normalised) angular frequency $\omega_{0} \in[0, \pi[$. In practice, electrical signals may be corrupted by harmonic content [21]. These frequency components are introduced by large nonlinear loads [21], [22]. Using these properties, the $n^{\text {th }}$ sample on the $m^{t h}$ phase $(m=0,1,2)$ can be expressed by [23]

$$
x_{m}[n]=\sum_{l=1}^{L} a_{l} \cos \left(l \omega_{0} n+\varphi_{l}-\frac{2 m l \pi}{3}\right)+b_{m}[n] .
$$

where $a_{l}$ and $\varphi_{l}$ correspond to the amplitude and initial phase of the $l^{\text {th }}$ harmonic component $(l=1,2, \cdots L)$ and $b_{m}[n]$ is the additive noise. Note that this signal model corresponds to the one described in the IEEE Standard 1459 for three-Phase nonsinusoidal balanced systems (see [23, Section 3.2.3]). In this paper, the problem of interest is to estimate $\omega_{0}$ from $N$ observations of the three-phase signal. Using matrix notation, the three-phase signal can be expressed as

$$
\mathbf{x}=\mathbf{G}\left(\omega_{0}\right) \mathbf{s}+\mathbf{b}
$$

where

- $\mathbf{x}$ and $\mathbf{b}$ are $3 N \times 1$ column vector which are defined respectively as

$$
\begin{aligned}
& \mathbf{x} \triangleq\left[x_{0}[0], \cdots, x_{0}[N-1], \cdots, x_{2}[0], \cdots, x_{2}[N-1]\right]^{T} \\
& \mathbf{b} \triangleq\left[b_{0}[0], \cdots, b_{0}[N-1], \cdots, b_{2}[0], \cdots, b_{2}[N-1]\right]^{T}
\end{aligned}
$$

where $(.)^{T}$ denotes the matrix transpose.

- $\mathbf{s}$ is a $2 L \times 1$ vector containing the amplitude and initial phase. This vector is defined as $\mathbf{s} \triangleq$ $\left[a_{1} \cos \left(\varphi_{1}\right), a_{1} \sin \left(\varphi_{1}\right), \cdots, a_{L} \cos \left(\varphi_{L}\right), a_{L} \sin \left(\varphi_{L}\right)\right]^{T}$.

- $\mathbf{G}\left(\omega_{0}\right)$ is a $3 N \times 2 L$ matrix that is defined as

$$
\mathbf{G}\left(\omega_{0}\right) \triangleq\left[\begin{array}{llll}
\mathbf{G}_{0}\left(1, \omega_{0}\right) & \mathbf{G}_{0}\left(2, \omega_{0}\right) & \cdots & \mathbf{G}_{0}\left(L, \omega_{0}\right) \\
\mathbf{G}_{1}\left(1, \omega_{0}\right) & \mathbf{G}_{1}\left(2, \omega_{0}\right) & \cdots & \mathbf{G}_{1}\left(L, \omega_{0}\right) \\
\mathbf{G}_{2}\left(1, \omega_{0}\right) & \mathbf{G}_{2}\left(2, \omega_{0}\right) & \cdots & \mathbf{G}_{2}\left(L, \omega_{0}\right)
\end{array}\right]
$$

where $\mathbf{G}_{m}\left(l, \omega_{0}\right) \triangleq\left[\Re e\left[\mathbf{a}_{m}^{T}\left(l, \omega_{0}\right)\right],-\Im m\left[\mathbf{a}_{m}^{T}\left(l, \omega_{0}\right)\right]\right]$ is a $N \times 2$ matrix and $\mathbf{a}_{m}\left(l, \omega_{0}\right)$ is defined as

$$
\mathbf{a}_{m}\left(l, \omega_{0}\right) \triangleq e^{-j \frac{2 \pi l m}{3}} \times\left[1, e^{2 l \omega_{0}}, \cdots, e^{l \omega_{0}(N-1)}\right]
$$

From a statistical point of view, the goal of this study is to estimate $\omega_{0}$ from $\mathbf{x}$ when $\mathbf{s}$ and $\mathbf{b}$ are unknown. To achieve this goal, we make the following assumptions:

- AS1) The number of components $L$ is known.

- AS2) The signal on each phase is corrupted by an additive white gaussian noise with zero mean and covariance $\sigma^{2}$, i.e. $\mathbf{b}=\mathcal{N}\left(\mathbf{0}, \sigma^{2} \mathbf{I}_{3 N}\right)$, where $\mathbf{I}_{3 N}$ corresponds to the identity matrix of size $3 N \times 3 N$.

- AS3) The number of samples, $N$, is greater than $(2 L+$ 1) $/ 3$.

Note that if $L$ is unknown, it can be estimated with information criteria techniques [24]. Concerning AS2), this assumption is motivated by the Central Limit Theorem.

\section{MAXimum Likelihood Estimation}

In this section, we derive the Maximum Likelihood Estimator (MLE) of the fundamental frequency. Under assumption AS2), the MLE corresponds to the least squares estimator. The estimate of $\omega_{0}$ and $\mathbf{s}$ are therefore obtained by minimizing

$$
\|\mathbf{x}-\mathbf{G}(\omega) \mathbf{s}\|^{2}
$$

with respect to $\omega$ and $\mathbf{s}$, where $\|\cdot\|^{2}$ corresponds to the vector norm. This nonlinear problem is a separable least squares problem. Consequently, it can be shown that the estimate of $\omega_{0}$ is obtained by maximizing [25, Section 8.9]

$$
\mathcal{C}(\omega)=\mathbf{x}^{T} \mathbf{G}(\omega)\left(\mathbf{G}^{T}(\omega) \mathbf{G}(\omega)\right)^{-1} \mathbf{G}^{T}(\omega) \mathbf{x}
$$

In the general case, the maximization of (4) must be performed by a grid-search algorithm or iterative techniques. Another issue is the computation of the inverse of $\mathbf{G}^{T}(\omega) \mathbf{G}(\omega)$ in (4). Using the expression of $\mathbf{G}(\omega)$, this matrix can be decomposed as

$$
\mathbf{G}^{T}(\omega) \mathbf{G}(\omega)=\left[\begin{array}{cccc}
\mathbf{P}_{1,1}(\omega) & \mathbf{P}_{1,2}(\omega) & \cdots & \mathbf{P}_{1, L}(\omega) \\
\mathbf{P}_{2,1}(\omega) & \mathbf{P}_{2,2}(\omega) & \cdots & \mathbf{P}_{2, L}(\omega) \\
\vdots & & \ddots & \vdots \\
\mathbf{P}_{L, 1}(\omega) & \mathbf{P}_{L, 2}(\omega) & \cdots & \mathbf{P}_{L, L}(\omega)
\end{array}\right]
$$

where

$$
\mathbf{P}_{u, v}(\omega) \triangleq \sum_{m=0}^{2} \mathbf{G}_{m}^{T}(u, \omega) \mathbf{G}_{m}(v, \omega)
$$

Although $\mathbf{P}_{u, v}(\omega)$ has a simple form, the inverse of $\mathbf{G}^{T}(\omega) \mathbf{G}(\omega)$ is difficult, if not impossible, to obtain analytically. However, in some particular cases, the structure of $\mathbf{P}_{u, v}(\omega)$ can be simplified and the inverse of $\mathbf{G}^{T}(\omega) \mathbf{G}(\omega)$ derived analytically.

\section{A. Single sinusoidal component $(L=1)$}

In the ideal case, the electrical signal contains only a sinusoidal component. Setting $L=1$ in (5), we obtain

$$
\mathbf{G}^{T}(\omega) \mathbf{G}(\omega)=\mathbf{P}_{1,1}(\omega)=\frac{3 N}{2} \mathbf{I}_{2} .
$$

Using this result, the cost function in (4) can be expressed as

$$
\mathcal{C}(\omega)=\frac{2}{3 N}\left\|\mathbf{G}^{T}(\omega) \mathbf{x}\right\|^{2}
$$

This expression can be simplified using the definition of $\mathbf{G}(\omega)$ and $\mathbf{x}$. By introducing the complex number $j^{2}=-1$, we obtain

$$
\mathcal{C}(\omega)=\frac{2}{3 N}\left|\sum_{n=0}^{N-1} \sum_{m=0}^{2} x_{m}[n] e^{-j\left(\omega n-\frac{2 m \pi}{3}\right)}\right|^{2}
$$

where |.| corresponds to the complex modulus.

Theorem 1: When $L=1$, the MLE of the fundamental frequency is obtained by maximizing

$$
P_{y_{1}}(\omega)=\frac{1}{N}\left|\sum_{n=0}^{N-1} y_{1}[n] e^{-j \omega n}\right|^{2}
$$


where $y_{1}[n] \triangleq \sum_{m=0}^{2} x_{m}[n] e^{\frac{2 j m \pi}{3}}$ is called the (instantaneous) positive sequence component.

Theorem 1 shows that the MLE of $\omega$ is given by the highest peak of the periodogram of the positive sequence component. We can note that the three-phase MLE is closely related to the MLE proposed by Rife [26]. Indeed, the main difference between these two estimators is the analysed signal. Specifically, the estimator proposed by Rife is based on the analytic signal whereas our estimator uses the positive sequence component.

\section{B. Estimation for $N \gg 1$}

For $N \gg 1$, it can be shown that

$$
\mathbf{P}_{u, v}(\omega) \approx \frac{3 N \delta(u-v)}{2} \mathbf{I}_{2}
$$

where $\delta(u)$ corresponds to the Kronecker delta. Using (11) in (5), we obtain

$$
\mathbf{G}^{T}(\omega) \mathbf{G}(\omega) \approx \frac{3 N}{2} \mathbf{I}_{2 L}
$$

Using this approximation in (4) and the definition of $\mathbf{G}(\omega)$, the cost function can be simplified as

$$
\mathcal{C}(\omega) \approx \frac{2}{3 N} \sum_{l=1}^{L}\left|\sum_{n=0}^{N-1} y_{l}[n] e^{-j l \omega n}\right|^{2}
$$

where $y_{l}[n] \triangleq\left[1 e^{2 j \pi l / 3} e^{4 j \pi l / 3}\right] x[n]$. The components $y_{l}[n]$ have a particular structure since $y_{l+3}[n]=y_{l}[n]$. To simplify the cost function, we make use of the Fortescue transform, which is defined as

$$
\left[\begin{array}{l}
y_{0}[n] \\
y_{1}[n] \\
y_{2}[n]
\end{array}\right] \triangleq\left[\begin{array}{ccc}
1 & 1 & 1 \\
1 & e^{2 j \pi / 3} & e^{4 j \pi / 3} \\
1 & e^{-2 j \pi / 3} & e^{-4 j \pi / 3}
\end{array}\right]\left[\begin{array}{l}
x_{0}[n] \\
x_{1}[n] \\
x_{2}[n]
\end{array}\right]
$$

where $y_{0}[n], y_{1}[n]$ and $y_{2}[n]$ are called the zero, positive and negative sequence components, respectively. This transform has been introduced by Fortescue in 1918 to simplify the analysis of unbalanced polyphase systems [27], [28]. Using (14) in (13), we obtain the following theorem.

Theorem 2: For $N \gg 1$, the MLE of the fundamental frequency is obtained by maximizing

$$
S(\omega)=\sum_{l=1}^{L}\left(\frac{1}{N}\left|\sum_{n=0}^{N-1} y_{l \bmod (3)}[n] e^{-j l \omega n}\right|^{2}\right)
$$

where $\bmod ($.$) is the modulo operator.$

Theorem 2 shows that the cost function corresponds to a cumulated periodogram involving the periodogram of $y_{0}[n]$, $y_{1}[n]$ and $y_{2}[n]$. It is interesting to note that the Fortescue transform separates the harmonic content of the three-phase signals. Indeed, for a particular harmonic order, only one sequence component contributes to the cost function. Specifically, information about the harmonics of order $l=3 q$, $l=3 q+1$ and $l=3 q+2(q \in \mathbb{N})$ are respectively carried by the zero, positive and negative sequence components.

\section{CRAmer RAO BOUND}

A natural criterion to assess the performance of an estimator is the mean square error (MSE). The MSE of $\widehat{\omega}$ is defined as $M S E[\widehat{\omega}]=\operatorname{bias}^{2}(\widehat{\omega})+\operatorname{var}(\widehat{\omega})$, where $\operatorname{bias}(\widehat{\omega})$ and $\operatorname{var}(\widehat{\omega})$ correspond to the bias and variance of the estimator. The variance of any unbiased estimator of the fundamental frequency, $\widehat{\omega}$, is bounded by [25]

$$
\operatorname{var}(\widehat{\omega}) \geq C R B(\widehat{\omega})
$$

where $C R B(\widehat{\omega})$ corresponds to the Cramer Rao Bound of $\widehat{\omega}$. Let us define $\theta \triangleq\left[a_{1}, \varphi_{1}, \cdots, a_{L}, \varphi_{L}, \omega\right]^{T}$ the $2 L+1$ vector containing the unknown parameters. The CRB of $\omega$ is given by

$$
C R B(\widehat{\omega})=\left[\mathbf{F}(\theta)^{-1}\right]_{2 L+1,2 L+1}
$$

where $\mathbf{F}(\theta)^{-1}$ is the inverse of the Fisher information matrix and $[.]_{i j}$ corresponds to the element located at the $i^{\text {th }}$ row and the $j^{t h}$ column. As $\mathbf{b}[n] \sim \mathcal{N}\left(0, \sigma^{2} \mathbf{I}_{3 N}\right)$, the $i j^{t h}$ element of the Fisher Information Matrix is given by [25]

$$
[\mathbf{F}(\theta)]_{i j} \triangleq \frac{1}{\sigma^{2}}\left(\frac{\partial \mathbf{G}(\omega) \mathbf{s}}{\partial \theta_{i}}\right)^{T}\left(\frac{\partial \mathbf{G}(\omega) \mathbf{s}}{\partial \theta_{i}}\right)
$$

Using the definitions of $\mathbf{G}(\omega)$ and $\mathbf{s}$, we obtain

$$
\begin{aligned}
\frac{\partial \mathbf{G}(\omega) \mathbf{s}}{\partial a_{l}}=\left[\begin{array}{l}
\mathbf{G}_{0}(l, \omega) \\
\mathbf{G}_{1}(l, \omega) \\
\mathbf{G}_{2}(l, \omega)
\end{array}\right]\left[\begin{array}{c}
\cos \left(\varphi_{l}\right) \\
\sin \left(\varphi_{l}\right)
\end{array}\right] \\
\frac{\partial \mathbf{G}(\omega) \mathbf{s}}{\partial \varphi_{l}}=\left[\begin{array}{l}
\mathbf{G}_{0}(l, \omega) \\
\mathbf{G}_{1}(l, \omega) \\
\mathbf{G}_{2}(l, \omega)
\end{array}\right]\left[\begin{array}{c}
-a_{l} \sin \left(\varphi_{l}\right) \\
a_{l} \cos \left(\varphi_{l}\right)
\end{array}\right] \\
\frac{\partial \mathbf{G}(\omega) \mathbf{s}}{\partial \omega}=\left[\begin{array}{lll}
\mathbf{H}_{0}(1, \omega) & \ldots & \mathbf{H}_{0}(L, \omega) \\
\mathbf{H}_{1}(1, \omega) & \ldots & \mathbf{H}_{1}(L, \omega) \\
\mathbf{H}_{2}(1, \omega) & \ldots & \mathbf{H}_{2}(L, \omega)
\end{array}\right] \mathbf{s}
\end{aligned}
$$

where $\mathbf{H}_{m}(u, \omega) \triangleq-u\left[\Im m\left[\mathbf{D} \mathbf{a}_{m}^{T}(u, \omega)\right] \Re e\left[\mathbf{D a}_{m}^{T}(u, \omega)\right]\right]$ and $\mathbf{D}=\operatorname{diag}([0,1,2, \cdots,(N-1))$. In the general case, the inverse of the Fisher Information Matrix is difficult to obtain analytically. However, we show in the next subsections that analytical forms can be derived when $L=1$ and $N \rightarrow \infty$. For the sake of comparison, the Signal to Noise Ratio (SNR) is defined in the next subsections as

$$
\rho \triangleq \frac{1}{2 \sigma^{2}} \sum_{l=1}^{L} a_{l}^{2}
$$

\section{A. Closed form expression for $L=1$}

For $L=1$, the Fisher Information Matrix can be expressed as

$$
\mathbf{F}(\theta)=\frac{3}{\sigma^{2}}\left[\begin{array}{ccc}
\frac{N}{2} & 0 & 0 \\
0 & \frac{a_{1}^{2} N}{2} & \frac{a_{1}^{2}}{2} \sum_{n=0}^{N-1} n \\
0 & \frac{a_{1}^{2}}{2} \sum_{n=0}^{N-1} n & \frac{a_{1}^{2}}{2} \sum_{n=0}^{N-1} n^{2}
\end{array}\right]
$$


The CRB of $\omega$ is derived from the inverse of $\mathbf{F}(\theta)$. After some manipulations, the CRB for sinusoidal signals, denoted $C R B_{1}(\widehat{\omega})$, reduces to

$$
C R B_{1}(\widehat{\omega})=\frac{4}{N\left(N^{2}-1\right) \rho} .
$$

Equation (24) shows that the CRB depends on the sample length, $N$, and Signal to Noise Ratio, $\rho$. Specifically, the CRB decreases as $\rho$ or $N$ increases. Furthermore it shows that the CRB is highly sensitive to $N$ since this bound decreases as $1 /\left(N^{3}\right)$. It is interesting to compare this CRB with that obtained for single-phase systems [25, Example 3.14]. First, we note that the CRB expression in (24) is exact for all $N$ while the expression provided in [25] holds asymptotically. Next, we observe that the CRB of $\omega$ for three-phase systems is three times smaller than that obtained for single-phase systems. This result can be generalized to $M$-phase system and demonstrates the benefit of using the multidimensional nature of electrical signals for frequency estimation.

\section{B. Asymptotic expression for $N \gg 1$}

For $N \gg 1$, the Fisher Information matrix can be decomposed as follows

$$
\mathbf{F}(\theta)=\frac{1}{\sigma^{2}}\left[\begin{array}{ccccc}
\mathbf{K}_{11}(\omega) & 0 & \cdots & 0 & \mathbf{f}_{1}(\omega) \\
0 & \mathbf{K}_{22}(\omega) & \ddots & \vdots & \vdots \\
\vdots & \ddots & \ddots & 0 & \vdots \\
0 & \cdots & 0 & \mathbf{K}_{L L}(\omega) & \mathbf{f}_{L}(\omega) \\
\mathbf{f}_{1}^{T}(\omega) & \mathbf{f}_{2}^{T}(\omega) & \cdots & \mathbf{f}_{L}^{T}(\omega) & t(\omega)
\end{array}\right]
$$

where

$$
\begin{aligned}
\mathbf{K}_{u u}(\omega) & =\frac{3 N}{2}\left[\begin{array}{cc}
1 & 0 \\
0 & a_{u}^{2}
\end{array}\right] \\
\mathbf{f}_{u}(\omega) & =\frac{3 u a_{u}^{2} N^{2}}{4}\left[\begin{array}{l}
0 \\
1
\end{array}\right] \\
t(\omega) & =\frac{N^{3}}{2} \sum_{l=1}^{L} l^{2} a_{l}^{2}
\end{aligned}
$$

Using the result on the inverse of a partitioned matrix, the asymptotic $\mathrm{CRB}$, denoted $C R B_{\infty}[\widehat{\omega}]$, can be expressed as

$$
C R B_{\infty}(\widehat{\omega})=\frac{4 \beta}{N^{3} \rho}
$$

where $\beta \leq 1$ is defined as

$$
\beta \triangleq \frac{\sum_{l=1}^{L} a_{l}^{2}}{\sum_{l=1}^{L} l^{2} a_{l}^{2}} .
$$

From (27), we observe that the CRB depends on the signal length, SNR and parameter $\beta$. Comparing the asymptotic bound in (27) with that derived in [29], we observe that the CRB for three-phase systems is three times smaller than that obtained for single-phase systems. Comparing the asymptotic bound with that obtained for sinusoidal signals, we observe that $C R B_{\infty}(\widehat{\omega}) \approx \beta C R B_{1}(\widehat{\omega})$. As $\beta \leq 1$, it follows that the
CRB for harmonic signals is $1 / \beta$ times smaller than that obtained for sinusoidal signals. This statement demonstrates the utility of using the harmonic content for frequency estimation.

\section{Simulation Results}

In this section, we evaluate the performance of the proposed frequency estimators. Specifically, we focus on three estimators, namely the exact MLE in (4), the sinusoidal MLE in (10), and the approximated MLE in (15). For sake of brevity, we refer to these technique as $M L E, M L E_{1}$, and $M L E_{\infty}$, respectively. These estimators are compared with the singlephase MLE [10] and with the Cramer Rao Bounds reported in Section IV. For each estimator, the maximisation step is performed using the Nelder-Mead simplex algorithm [30]. This algorithm is initialized at the nominal fundamental frequency, i.e. $\widehat{w}=2 \pi \times 50 / F_{s} \mathrm{rad} / \mathrm{s}$, and the termination tolerance is set to $10^{-8}$. The frequency estimators are tested using signals modeled by (1) under different signal length, $N$. The performance are evaluated in terms of Mean Square Error (MSE). The MSE is estimated using $K=1000$ Monte Carlo Trials by

$$
M S E \approx \frac{1}{K} \sum_{k=0}^{K-1}\left(\omega_{0}-\widehat{\omega}\right)^{2}
$$

where $\omega_{0}$ is the (true) fundamental frequency and $\widehat{\omega}$ corresponds to the frequency estimate. In each simulation, the sampling frequency and the fundamental frequency are set to $F_{s}=1 \mathrm{kHz}$ and $\omega_{0}=2 \pi \times(51.5) / F_{s} \mathrm{rad} / \mathrm{s}$, respectively.

\section{A. Estimation for $L=1$}

In this subsection, we evaluate the performance for sinusoidal signals. In each simulation, parameters are fixed to $L=1, a_{1}=1, \varphi=0.3$ and $S N R=10 \mathrm{~dB}$. Figure 1 presents the performance of the three-phase and single-phase MLE. We note that the three-phase MLE achieves the CRB. Furthermore, we observe that the three-phase estimator outperforms the single-phase estimator, whatever the number of samples. Specifically, the former decreases the MSE by a factor of three, which is consistent with the conclusions of subsection IV-A.

\section{B. Estimation for $L \geq 1$}

In this subsection, we present the performance of $M L E$, $M L E_{1}$ and $M L E_{\infty}$ for harmonic signals with $L=4$. Simulation parameters are given in Table I. Figure 2 displays the MSE versus the data-length at $S N R=10 \mathrm{~dB}$. We observe that the exact and asymptotic CRBs have similar values whatever the signal length. Furthermore, among the considered estimators, we note that the exact MLE is the only one that achieves the CRB at high SNRs. As compared to single-phase and $M L E_{1}$ estimators, the exact MLE significantly decreases the MSE. Concerning the approximated MLE, $M L E_{\infty}$, we see that the performances highly depend on the signal length $N$. Indeed, the performances tend to those of the exact MLE for $N \rightarrow \infty$. In addition, we observe that the MSEs are broadly similar for $N \approx 2 k \pi / \omega(k \in \mathbb{N})$. This behaviour comes from the fact that the equality in (11) is attained for these particular values of $N$. 


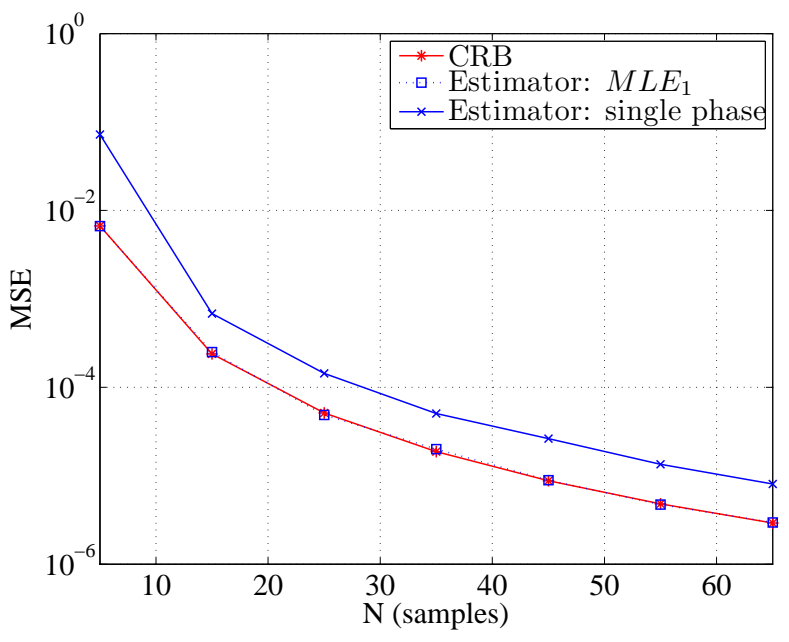

Fig. 1: MSE versus signal length for sinusoidal signals $(L=1$, $S N R=10 \mathrm{~dB})$.

TABLE I: Simulation parameters $(L=4)$.

\begin{tabular}{|c|cccccccc|}
\hline Parameter & $a_{1}$ & $a_{2}$ & $a_{3}$ & $a_{4}$ & $\varphi_{1}$ & $\varphi_{2}$ & $\varphi_{3}$ & $\varphi_{4}$ \\
\hline Value & 1 & 0.1 & 0.105 & 0.366 & 0.052 & 0.1 & 0.4 & 0.5 \\
\hline
\end{tabular}

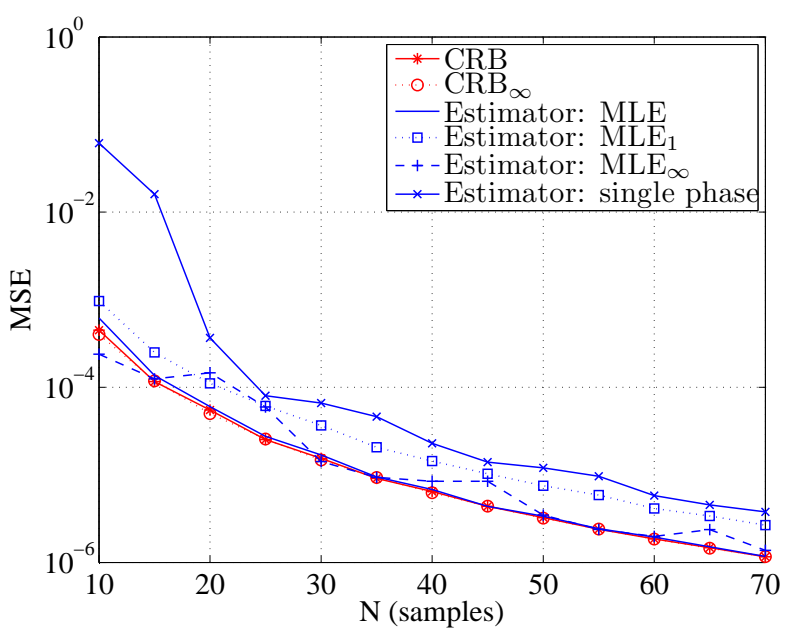

Fig. 2: MSE versus signal length, $N$, for harmonic signals $(L=4, \beta=0.5, S N R=10 \mathrm{~dB})$.

\section{Experimental signals}

In this subsection, the frequency estimator $M L E$ is applied to experimental data. Experimental signals come from the DOE/EPRI National Database of Power System Events. The sampling frequency is equal to $F_{e}=15360 \mathrm{~Hz}$. The analysed signals are presented in Figures 3 and 4. These signals correspond to the events 3127 and 3163 . The fundamental frequency is estimated using the exact three-phase MLE estimator with $L=3$. Frequency estimates obtained with the single phase MLE $(L=3)$ and root MUSIC are also shown for comparison.

Figure 3 displays the estimated fundamental frequencies versus $N$ for the event 3127 . We observe that the three estima-

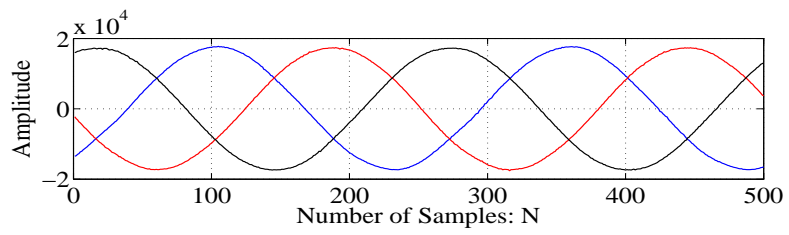

(a) Experimental Signal.

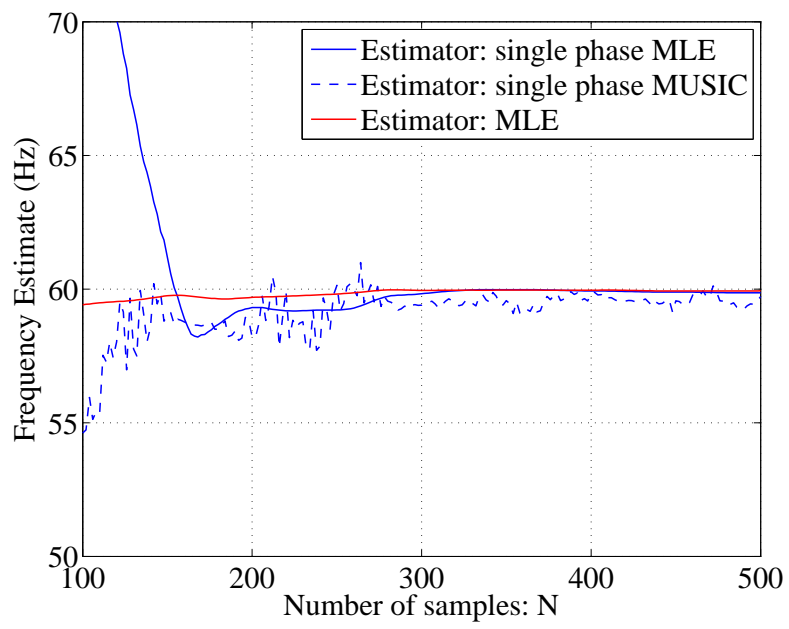

(b) Frequency estimation versus the number of samples $N$.

Fig. 3: Event 3127: Estimation of the fundamental frequency.

tors converge to the same value, $\widehat{f_{0}}=59.9 \mathrm{~Hz}$, when $N>500$. However, we see that the exact MLE converges more rapidly than the single-phase MLE and root MUSIC. In particular, the exact MLE is the only estimator that provides accurate results even for a small number of samples. Specifically, this estimator converges roughly after 275 samples $(\approx 1$ cycle $)$.

As shown in Figure 4, the event 3163 introduces a moderate amount of three-phase unbalance in the voltage signal. Although the proposed estimator is designed for balanced systems, we note that the single-phase and proposed estimator lead to the same frequency estimate for $N>500$ samples $(\approx$ 2 cycles). Nevertheless, the three-phase MLE exhibits extra oscillations due to the unbalanced conditions. We can note that these oscillations significantly increase the estimator's settling time. Under unbalanced conditions, it is clear that better estimates could be obtained by treating the unbalance parameters as nuisance parameters.

\section{CONCLUSions}

In this paper, we have focused on the Maximum Likelihood estimation of the fundamental frequency in threephase balanced systems. A new estimator that jointly uses the multidimensional and harmonic structure of electrical signals has been proposed. For sinusoidal signals, this estimator is obtained by maximizing the periodogram of the positive sequence component. For harmonic signals, the estimator is obtained by maximizing a cumulated periodogram of the zero, positive and negative components. The analysis of the Cramer Rao Bound has clearly demonstrated the benefit of using 


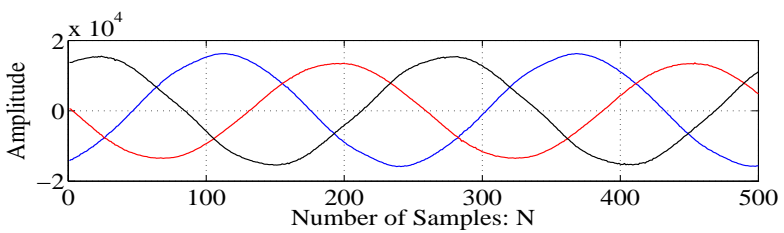

(a) Experimental Signal.

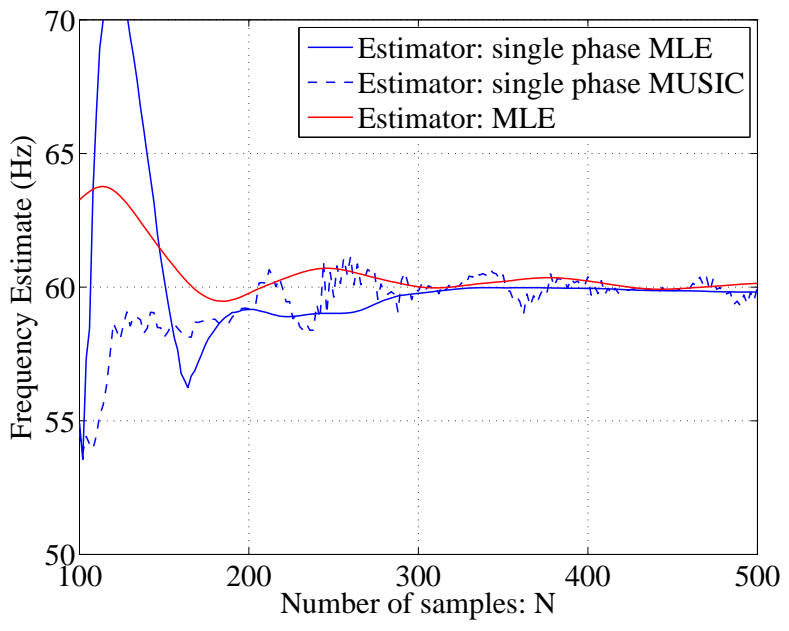

(b) Frequency estimation versus the number of samples $N$.

Fig. 4: Event 3163: Estimation of the fundamental frequency.

the multidimensional and harmonic structure for frequency estimation. Indeed, the use of the multidimensional nature of electrical signals allows to decreases the CRB by of a factor of 3 , and the use of the harmonic structure by a factor of $1 / \beta$.

\section{REFERENCES}

[1] A. Kwasinski, "Signal processing in the electrification of vehicular transportation: Techniques for electric and plug-in hybrid electric vehicles on the smart grid," IEEE Signal Processing Magazine, vol. 29, no. 5, pp. 14-23, 2012.

[2] S. M. Kay and S. L. Marple Jr, "Spectrum analysis - a modern perspective," Proceedings of the IEEE, vol. 69, no. 11, pp. 1380-1419, 1981.

[3] P. Stoica and R. L. Moses, Introduction to Spectral Analysis. Upper Saddle River, NJ: Prentice-Hall, 1997.

[4] M. M. Begovic, P. M. Djuric, S. Dunlap, and A. G. Phadke, "Frequency tracking in power networks in the presence of harmonics," IEEE Transactions on Power Delivery, vol. 8, no. 2, pp. 480-486, 1993.

[5] M. Karimi-Ghartemani and M. Iravani, "A nonlinear adaptive filter for online signal analysis in power systems: applications," IEEE Transactions on Power Delivery, vol. 17, no. 2, pp. 617-622, 2002.

[6] T. Lobos, Z. Leonowicz, J. Rezmer, and P. Schegner, "High-resolution spectrum-estimation methods for signal analysis in power systems," IEEE Transactions on Instrumentation and Measurement, vol. 55, no. 1, pp. 219-225, 2006.

[7] A. Bracale, G. Carpinelli, D. Lauria, Z. Leonowicz, T. Lobos, and J. Rezmer, "On some spectrum estimation methods for analysis of nonstationary signals in power systems. part i. theoretical aspects," in 11th International Conference on Harmonics and Quality of Power, 2004. IEEE, 2004, pp. 266-271.

[8] M. Kusljevic, "On LS-Based Power Frequency Estimation Algorithms," IEEE Transactions on Instrumentation and Measurement, vol. 62, no. 7, pp. 2020-2028, 2013.

[9] M. Mojiri, M. Karimi-Ghartemani, and A. Bakhshai, "Estimation of power system frequency using an adaptive notch filter," IEEE Transactions on Instrumentation and Measurement, vol. 56, no. 6, pp. 2470 2477, 2007.
[10] R. Chudamani, K. Vasudevan, and C. Ramalingam, "Real-time estimation of power system frequency using nonlinear least squares," IEEE Transactions on Power Delivery, vol. 24, no. 3, pp. 1021-1028, 2009.

[11] H. Li, P. Stoica, and J. Li, "Computationally efficient parameter estimation for harmonic sinusoidal signals," Signal Processing, vol. 80, no. 9, pp. 1937-1944, 2000.

[12] A. Pradhan, A. Routray, and A. Basak, "Power system frequency estimation using least mean square technique," IEEE Transactions on Power Delivery, vol. 20, no. 3, pp. 1812-1816, 2005.

[13] F. D. Freijedo, J. Doval-Gandoy, O. Lopez, and E. Acha, "A generic open-loop algorithm for three-phase grid voltage/current synchronization with particular reference to phase, frequency, and amplitude estimation," IEEE Transactions on Power Electronics, vol. 24, no. 1, pp. 94-107, 2009.

[14] V. Choqueuse, M. Benbouzid, Y. Amirat, and S. Turri, "Diagnosis of three-phase electrical machines using multidimensional demodulation techniques," IEEE Transactions on Industrial Electronics, vol. 59, no. 4, pp. 2014-2023, 2012.

[15] V. Choqueuse, A. Belouchrani, E. El Bouchikhi, and M. Benbouzid, "Estimation of Amplitude, Phase and Unbalance Parameters in Threephase Systems: Analytical Solutions, Efficient Implementation and Performance Analysis," IEEE Transactions on Signal Processing, vol. 62, no. 16, pp. 4064-4076, Aug 2014.

[16] Y. Chen and H. C. So, "Accurate parameter estimation for unbalanced three-phase system," The Scientific World Journal, vol. 2014, 2014.

[17] Y. Xia, S. C. Douglas, and D. P. Mandic, "Adaptive frequency estimation in smart grid applications: Exploiting noncircularity and widely linear adaptive estimators," IEEE Signal Processing Magazine, vol. 29, no. 5, pp. 44-54, 2012.

[18] D. H. Dini and D. P. Mandic, "Widely linear modeling for frequency estimation in unbalanced three-phase power systems," IEEE Transactions on Instrumentation and Measurement, vol. 62, no. 2, pp. 353-363, 2013.

[19] T. Routtenberg and L. Tong, "Joint frequency and phasor estimation under the kcl constraint," IEEE Signal Processing Letters, vol. 20, no. 6 , pp. 575-578, 2013.

[20] $\_$, "Joint frequency and phasor estimation in unbalanced three-phase power systems," in Acoustics, Speech and Signal Processing (ICASSP), 2014 IEEE International Conference on, May 2014, pp. 2982-2986.

[21] "IEEE standard for synchrophasor measurements for power systems," IEEE Std C37.118.1-2011 (Revision of IEEE Std C37.118-2005), pp. 1-61, Dec 2011.

[22] P. Top, M. Bell, E. Coyle, and O. Wasynczuk, "Observing the power grid: Working toward a more intelligent, efficient, and reliable smart grid with increasing user visibility," IEEE Signal Processing Magazine, vol. 29, no. 5, pp. 24-32, 2012.

[23] "IEEE Trial-Use Standard Definitions for the Measurement of Electric Power Quantities Under Sinusoidal, Non-sinusoidal, Balanced, Or Unbalanced Conditions," IEEE Std 1459-2000, pp. i-44, 2000.

[24] P. Stoica and Y. Selen, "Model-order selection: a review of information criterion rules,' IEEE Signal Processing Magazine, vol. 21, no. 4, pp. 36-47, 2004.

[25] S. Kay, Fundamentals of Statistical Signal Processing: Estimation Theory. Englewood Cliffs, NJ: Prentice-Hall signal processing series, 1993.

[26] D. Rife and R. Boorstyn, "Single-tone parameter estimation from discrete observations," IEEE Transactions on Information Theory, vol. 20, no. 5, pp. 591-598, 1974.

[27] C. Fortescue, "Method of symmetrical co-ordinates applied to the solution of polyphase networks," Transactions of the American Institute of Electrical Engineers, vol. XXXVII, no. 2, pp. 1027-1140, 1918.

[28] F. Furfari and J. Brittain, "Charles LeGeyt Fortescue and the method of symmetrical components," IEEE Industry Applications Magazine, vol. 8 no. 3, pp. 7-9, 2002.

[29] A. Nehorai and B. Porat, "Adaptive comb filtering for harmonic signal enhancement," IEEE Transactions on Acoustics, Speech and Signal Processing, vol. 34, no. 5, pp. 1124-1138, 1986.

[30] J. C. Lagarias, J. A. Reeds, M. H. Wright, and P. E. Wright, "Convergence properties of the nelder-mead simplex method in low dimensions," SIAM Journal on Optimization, vol. 9, no. 1, pp. 112-147, 1998. 